\title{
Chaos in Casting: An Approach to Shrinkage Porosity
}

\author{
Dieter Ott, \\ FEM, Schwibisch Gmiind, Germany
}

\begin{abstract}
Experimental data on the effects of various factors on the incidence of shrinkage porosity in cast gold jewellery items have been accumulated in recent years. Consideration of these data leads to the conclusion that certain of these effects are unpredictable, or 'chaotic'. Possible reasons for this are presented and available experimental data are discussed and illustrated in terms of this new approach to shrinkage porosity. Interactions between the effects of different factors are illustrated and discussed as also contributing to the unpredictability of results in some instances.
\end{abstract}

The incidence of shrinkage porosity in cast carat gold jewellery items is a continuing problem in jewellery fabrication and the effects upon it of various factors have received particular attention at the FEM. The resulting data have sometimes proved to be confusing and apparently inconsistent. The implications of this are discussed; it is apparent that a new and broader approach is needed for interpreting and drawing conclusions from the data. It has been concluded that the effects of certain factors are chaotic or unpredictable. Moreover, in some instances interactions between different factors complicate further interpretation of the results.

\section{Kinds of uncertainty and unpredictability}

The result of a technical process is always more or less uncertain. As a consequence of this, the measured values of the properties of a product such as a casting will deviate more or less from expected values.

Three things may happen:

(a) The deviations may be strictly related to variations in production conditions, $i e$ we are able to predict the properties directly by measuring and controlling these parameters (related deviations).

(b) The deviations may be influenced by many factors which can neither be detected nor measured separately. The effect of single factors may be very small, but together these factors cause a scattering of the properties which determine the quality of the product in a statistical or stochastic manner (stochastic deviations). (c) In the worst case the system (or product) displays chaotic behaviour. In this case predictions are impossible (chaotic behaviour):

Deviations also arise from interactions between different factors, and such interactions can make interpretation of results difficult.

\section{What is chaos?}

A system will behave chaotically if a small variation of input causes an unpredictable (and considerable) variation in output. This is appropriately illustrated using a mathematical model (see Figure 1). Thus in the mathematical function $\mathrm{X}_{\mathrm{i}+1}=\mathrm{R}^{*} \mathrm{x}_{\mathrm{i}}^{*}\left(1-\mathrm{x}_{\mathrm{i}}\right)$, values for $\mathrm{x}$ can be calculated with a running number, which

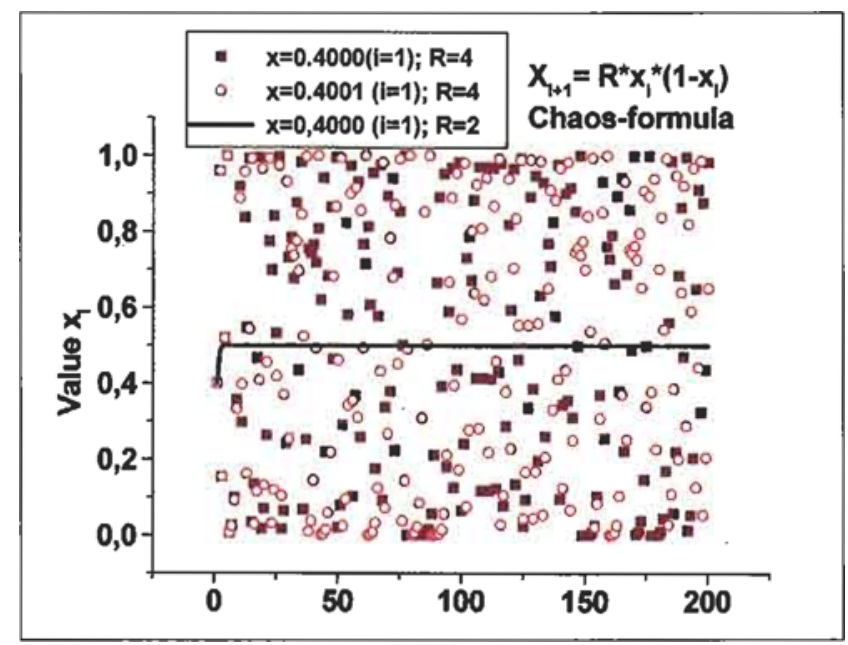

Figure 1 Demonstration of a chaotic process 
means that the value of each $\mathrm{x}$ depends on that of its predecessor. Thus, if we assume an initial value for $\boldsymbol{x}=$ 0.4 and a constant $\boldsymbol{R}=2$, the value for $\boldsymbol{x}$ will very quickly approximate to a constant and predictable value of 0.5 . If we change $R$ to 4 , the values scatter around a mean value of about 0.5 in an unpredictable way. A kind of (unpredictable) pattern can be recognized. It is sufficient to change the initial value for $\boldsymbol{x}$ by a very small extent and get a totally different pattern. The result is a model for a chaotic process: An extremcly small variation in input changes the output considerably.

\section{Chaos in science and technique: a principle of natural processes}

The very strange behaviour of a system, as demonstrated above in the case of a simple mathematical model, can also be recognized in natural or technical processes. Under certain conditions a small variation of initial values can change the output pattern completely and in an unforeseen way. A well known example is the growth of ice flowers on a window-pane. This example is very close to the situation which occurs on solidification of a melt.

\section{Factors operating during investment casting}

The quality of a cast item is significantly determined by the process of crystallization. First of all, crystallization depends on heat transfer. Heat transfer itself is determined by the temperature and thermal conductivity of the investment and melt, geometrical relations, mass and heat capacity, heat of solidification and so on. In principle, heat transfer might be regarded as calculable provided that all the necessary parameters are known or measurable. In practice, however, this is impossible. The best result that could be achieved is a rough approximation with a considerable scatrering. The scattering can be regarded as statistical because it is the result of a great number of small influences.

In addition, crystallization also depends on nucleation and crystal growth. Nucleation has an accidental component. Crystallization follows nucleation in a more or less chaotic way. Thus a very small accidental variation in nucleation will influence the crystallization process in a non-predictable way.

\section{The causes of shrinkage porosity}

Shrinkage porosity is caused by difficulties in the supply of molten metal needed to compensate for the sharp decrease of metal volume which occurs during solidification. Premature freezing of the sprue or thin cross-sectioned parts of the item is one reason for difficulties in melt supply. Another is the formation of a dendritic three dimensional network by the growing crystallites. The resistance to melt flow is considerably increased by this process.

The formation and the growth of a dendritic network, however, happens more or less chaotically, since nucleation is a random process and the growth of the network follows chaotic principles. This is also valid for formation of porosity because it is strongly connected with dendritic growth. Success or failure of measures for improving soundness of cast items can, therefore, be determined only by statistics. It is impossible to predict the incidence of porosity in a specified cast item.

These more or less theoretical conclusions receive support from practical experience gained in studies of shrinkage porosity.

\section{Factors influencing shrinkage porosity and general remarks}

Shrinkage porosity is influenced by :

- Temperatures (flask and melt)

- Shape of the cast item (stereometric relations, especially the surface/volume ratio and changes between thin and thick parts within a specified item).

- Casting method

- Thermal properties of the investment

- Properties of the alloy

The influence of temperature is considered in more detail in what follows. The shape of the casting has an important influence, too. However, an experimental investigation is difficult because there is no 'standard jewellery' with easily calculable stereometric relations. In our investigations we have therefore used as our 'standard' a ring consisting of a sphere (as the head) and a cylindrical shank. Sphere and shank diameters were varied within practical limits.

A basic factor for getting good casting quality is the composition of the alloy. For gold jewellery, casting alloys are made primarily to match fineness (caratage) and colour. Casting properties have not been so much of a criterion in the past. A recent project on shrinkage porosity addressed this problem to some extent and the results are presented below.

The effect of a specific factor has been found to depend very strongly on the influence of other parameters and this is a characteristic of casting. For example, an increase in flask temperature has different effects at low and high casting temperatures respectively. This is referred to as interaction. Interactions are very common and add to the 
complexity of understanding random behaviour and chaos effects on shrinkage porosity.

Another problem is that porosity is not uniformly distributed within a cast item. Different parts show different reactions to variations of an influencing factor. Therefore we were forced to make evaluations separately for different parts of castings.

For this purpose, different sections of castings such as sphere surface, sphere centre, changeover (between shank and sphere), shank and sprue (connection of shank with sprue) have been examined. Different parts were sometimes found to react in opposite directions. Improved quality of the sphere surface was found to increase porosity of changeover, for example.

\section{SUMMARY OF EXPERIMENTAL CONDITIONS}

\section{Alloys}

Table 1 gives some details of the alloy compositions used for this investigation and some of their characteristics.

\section{Temperatures}

Flask temperature was varied between 200 and $700^{\circ} \mathrm{C}$. The casting temperature was varied in three levels between $950^{\circ} \mathrm{C}$ and $1300^{\circ} \mathrm{C}$. The levels were chosen depending on alloys. Level low: about $100^{\circ} \mathrm{C}$, medium: about $200^{\circ} \mathrm{C}$ and high: about $300^{\circ} \mathrm{C}$ above liquidus temperature.

\section{Investment}

Only phosphate bonded investment was used to avoid interference by gas porosity

\section{Casting method}

A pressure assisted static casting method was used. The pressure difference was about 500 mbar (in most cases), the starting pressure 200 mbar argon.

\section{Cast items}

The stereometric simple item simulated a kind of ring consisting of a sphere and a cylindrical (in one case a flat) shank with varying diameters.

\section{Measurement of porosity and evaluation}

Porosity was measured by image analysis in micro sections. A new variable was introduced which takes into account simultaneously percentage of porosity, mean pore volume and scattering of these values. The new variable is simply called 'porosity'.

\section{PRESENTATION OF DATA}

The first task was to find out which factors have an effect on porosity and whether significant interactions exist between them. Variance analysis was used for this purpose. Evaluation was performed separately for different sections of the cast item. Figures 2 and 3 depict examples of the results obtained by this method. Factors marked at a distance from the centre of the circle have a highly significant influence on porosity. Marks in the centre indicate a less significant effect. Factors which are not marked within the circle do not influence porosity significantly. The factors are numbered. Main effects are labelled with name (abbreviated) and number. Twofold interactions are indicated as multipliers, eg $1 \times 2$ means that the influence of alloy on porosity depends on flask

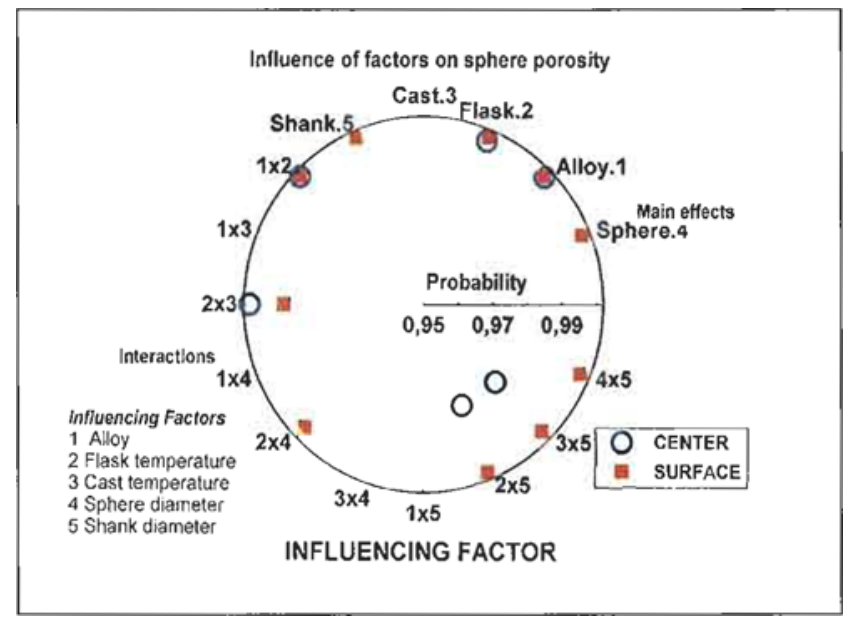

Figure 2 Influence of factors on the porosity of the sphere

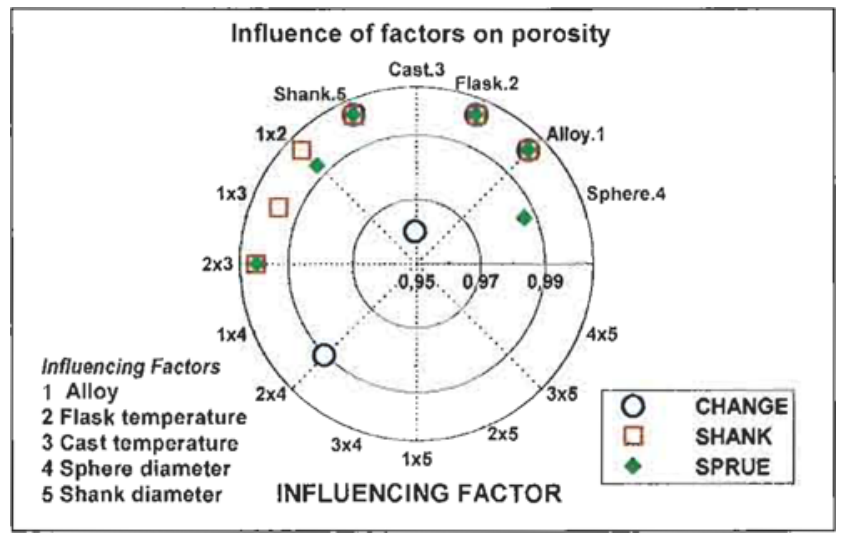

Figure 3 Influence of factors on porosity of changeover, shank and sprue 
Table 1 Alloy compositions and properties

$\begin{array}{cccccccc}\begin{array}{c}\text { Alloy } \\ \text { No }\end{array} & \text { Au \% } & \text { Ag \% } & \mathbf{C u} \% & \mathbf{Z n} \% & \begin{array}{c}\text { Solidus } \\ { }^{\circ} \mathbf{C}\end{array} & \begin{array}{c}\text { Liquidus } \\ { }^{\circ} \mathbf{C}\end{array} & \begin{array}{c}\text { Density } \\ \mathbf{8} / \mathbf{c m}^{\mathbf{3}}\end{array} \\ 1 & 58.50 & 30.00 & 11.50 & - & 841 & 901 & 13.92 \\ 2 & 58.50 & 17.00 & 19.50 & 5 & 794 & 833 & 13.52 \\ 3 & 75.00 & 16.00 & 9.00 & - & 905 & 933 & 15.45 \\ 4 & 75.00 & 2.00 & 22.00 & 1 & 897 & 917 & 15.45 \\ 5 & 75.00 & 14.00 & 9.00 & 2 & 865 & 903 & 15.36 \\ 6 & 91.66 & 6.20 & 2.14 & - & 1010 & 1035 & 18.00 \\ 7 & 91.66 & 2.14 & 6.20 & - & 959 & 982 & 17.80\end{array}$

temperature. Three fold interactions (and higher) are not considered.

To summarize, the alloy composition has the strongest effect on porosity regardless of the position in the casting. The melt temperature shows the weakest influence of all the main effects. There are many interactions. One of the most prominent is that the influence of alloy composition strongly depends on the flask temperature and this result will be examined in more detail later.

Although the casting temperature itself apparently has no significant effect, an interaction with flask temperature is very probable in most cases.

\section{GENERAL EFFECT OF ALLOY COMPOSITION}

Variance analysis shows only that there is a significant effect. No information can be given on its kind and magnitude. Further evaluation of the data is expected to provide this information, however.

Figure 4 gives a survey of the impact of alloy

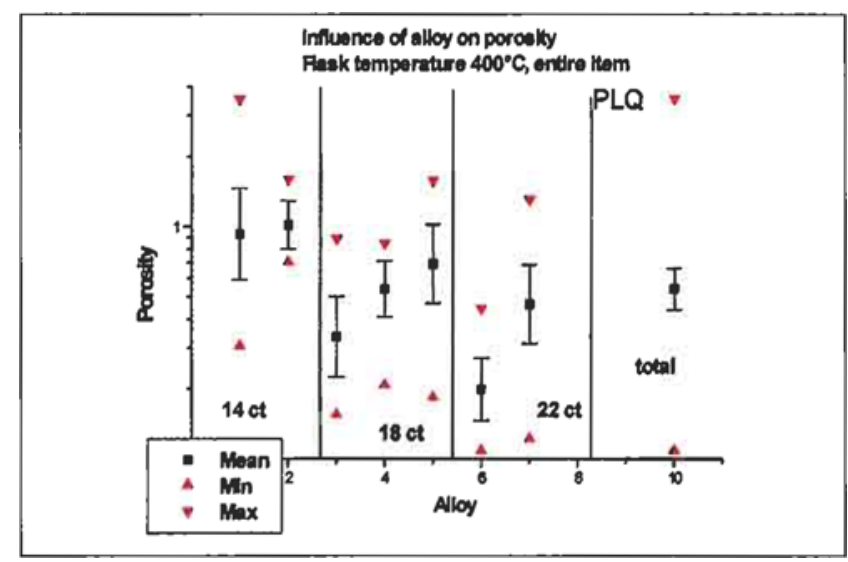

Figure 4 Influence of alloy on porosity of the entire item at $400^{\circ} \mathrm{C}$ flask temperature. composition on porosity. In these examples the porosity of the cast item as a whole (entire item) regardless of its shape was taken into account.

14 carat yellow gold castings had more porosity than all the other alloys under investigation independent of flask temperature. This result confirms experience gained in casting practice. In general, differences between alloys were more pronounced at low flask temperature. At $400^{\circ} \mathrm{C}$ flask temperature, 22 ct was superior to all other alloys.

\section{SURVEY OF THE INTERACTION BETWEEN ALLOY AND FLASK TEMPERATURE}

A survey of influence of flask temperature and alloy on porosity is given in Figure 5. Changes in temperature have different effects depending on alloy composition. The interaction is exceptionally prominent with $14 \mathrm{ct}$ gold. Not only the magnitude of the effects is of interest but also the principal function between porosity and flask temperature has to be considered

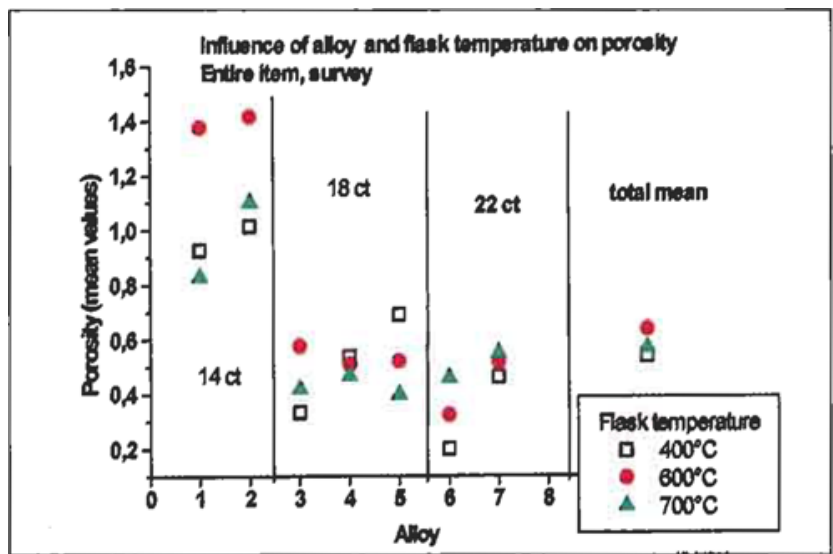

Figure 5 Survey of temperature effect on different alloys, entire item (for clarity only mean values are given) 


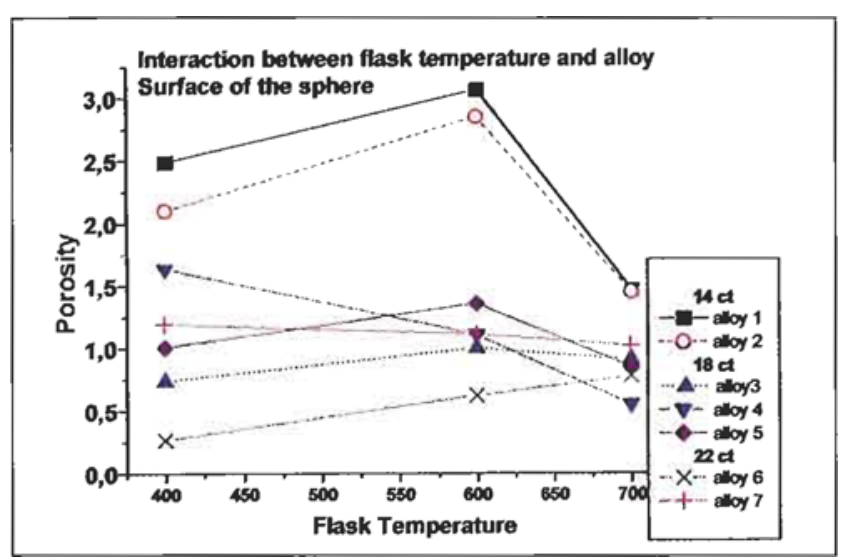

Figure 6 Temperature dependence on porosity as a function of alloy (surface of sphere)

and this strongly depends on alloy type (see Figure 6).

At a first glance the situation is confusing. However, on closer examination interesting facts are revealed. The $14 \mathrm{ct}$ alloys show a maximum of porosity at a flask temperature which is commonly used as a 'standard' $\left(600^{\circ} \mathrm{C}\right)$. The least porosity is observed at $700^{\circ} \mathrm{C}$. However, this is a temperature which should not be used with a gypsum bonded investment because of the danger of reaction with calcium sulfate.

The copper-rich 18 ct alloy (Alloy 4) has a decreasing tendency for porosity with increasing temperature. The silver-rich $18 \mathrm{ct}$ alloy (Alloy 3 ) shows a weak trend in the opposite direction. The dependence of the trend on copper concentration is even more clearly demonstrated with $22 \mathrm{ct}$ alloys. The porosity of the silver-rich Alloy 6 increases with flask temperature, whereas the porosity of copper-rich Alloy 7 decreases.

In order to obtain more reliable information, three $18 \mathrm{ct}$ alloys with varying silver/copper ratio were prepared. They are considered separately for more

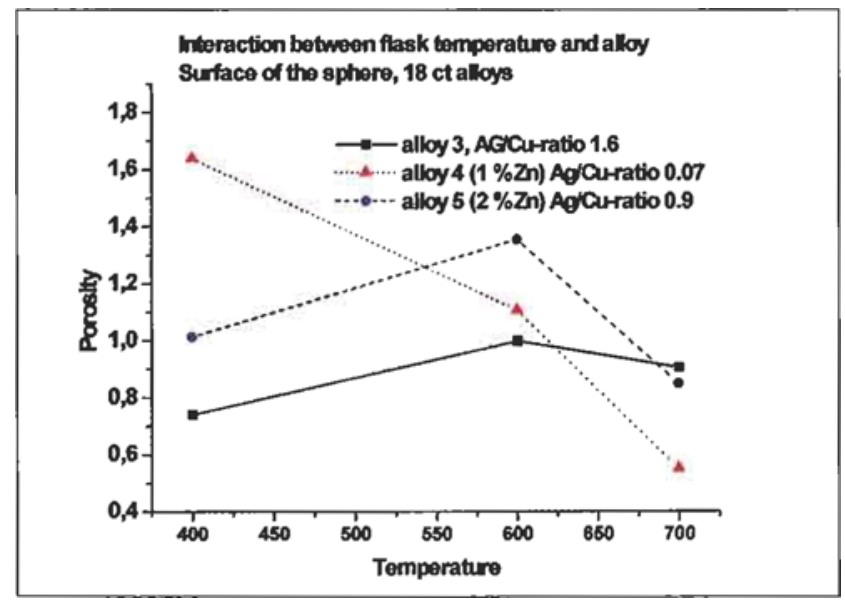

Figure 7 Temperature dependence on porosity of $18 \mathrm{ct}$ alloys (surface of sphere). clarity in Figure 7.

The porosity of Alloy 4 decreases sharply with increasing temperature. The alloy with the highest silver content shows a weak tendency in the opposite direction. At $600^{\circ} \mathrm{C}$ and above there is no significant dependence on temperature. The behaviour of the medium silver alloy lies between the other two alloys. At low temperature a relatively small increase in porosity, and between 600 and $700^{\circ} \mathrm{C}$ a more pronounced decrease occurs. As a consequence the porosity has a maximum at about $600^{\circ} \mathrm{C}$.

\section{INFLUENCE OF THE RATIO OF PRECIOUS METALS TO NON- PRECIOUS METALS ON THE POROSITY}

Figure 8 demonstrates the dependence of porosity on the ratio of precious to non-precious metals at a $400^{\circ} \mathrm{C}$ flask temperature (from a metallurgical point of view it was reasonable to use the atomic ratio).

There is a strong influence of the ratio on quality of the castings even if the great scattering of the values is taken into consideration (please note that a logarithmic scale is used).

At $600^{\circ} \mathrm{C}$ there is a decrease of porosity with the increase of precious to non-precious metal ratio. However, the values have a very large scatter. One reason could be that, in addition, the copper to silver ratio is very important at this temperature. At $700^{\circ} \mathrm{C}$ there is only a small effect, almost non-detectable with logarithmic scaling.

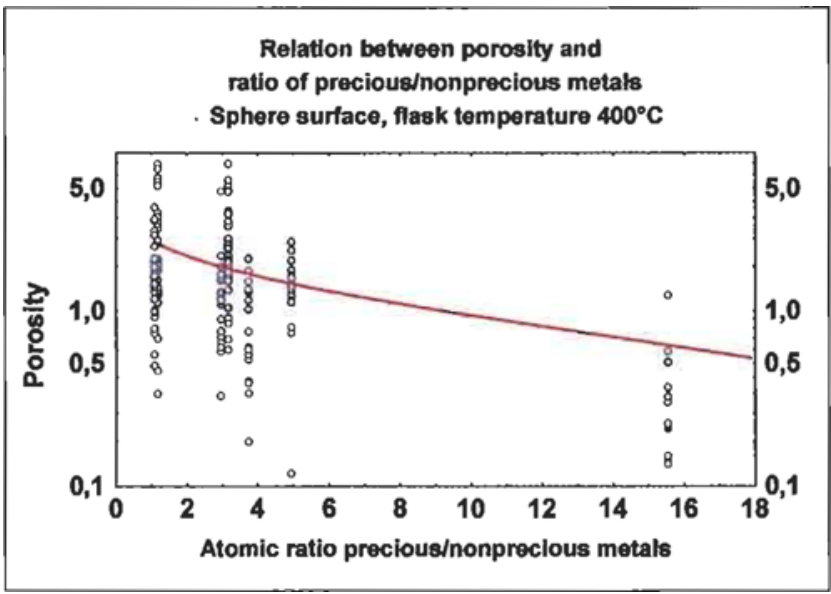

Figure 8 Influence of precious/non-precious metal ratio on porosity of sphere surface at $400^{\circ} \mathrm{C}$ 
Influence of silver/copper ratio and flask temperature on porosity of sphere surface, $18 \mathrm{ct}$ alloys

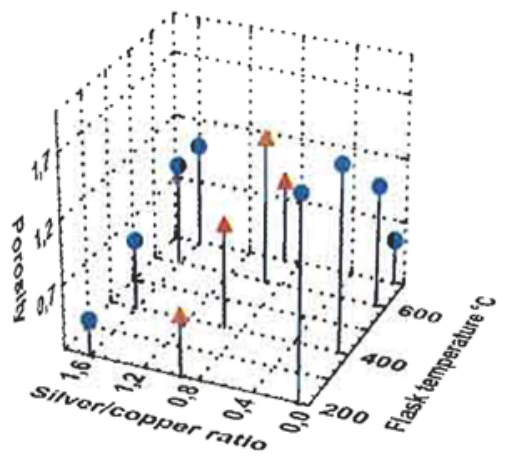

Figure 9 Influence of silver/copper ratio and flask temperature on porosity of sphere surface 18 ct alloys

Figure 9 indicates the effect of copper/silver ratio using $18 \mathrm{ct}$ alloys as an example. The figure demonstrates very clearly that each composition has its optimum flask temperature.

A correlation even exists between absolute copper concentration and porosity. However, the correlation is not very strong and scatter is considerable (many other influencing factors are not taken into account). Increasing the copper content increases the porosity.

\section{INFLUENCE OF OTHER PROPERTIES OF ALLOYS}

\section{Density}

Density shows a similar effect to that of copper concentration or metal ratio, since density is strongly related on metal ratio.

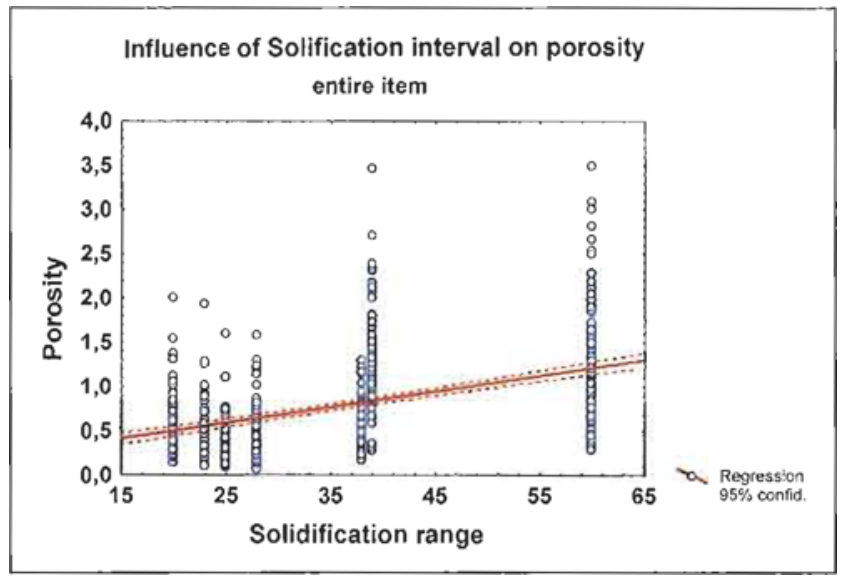

Figure 10 Influence of melting interval on porosity of the entire cast item

\section{Melting range}

The melting range correlates with porosity. The greater the melting range, the greater the porosity (Figure 10). This effect, however, is not very strong. It has to be noted that metal ratio and solidification range are connected. The increase of scattering with increasing melting interval is more remarkable. Obviously, quality is less consistent and more strongly influenced by casting conditions if the melting range is larger.

\section{THE ROLE OF CASTING TEMPERATURE}

Casting temperature is a parameter which can be changed easily within a limited range. However - as mentioned above - no significant principal effect on formation of shrinkage porosity could be established. On the other hand, a strong interaction with flask temperature was observed. Figure 11 provides an example.

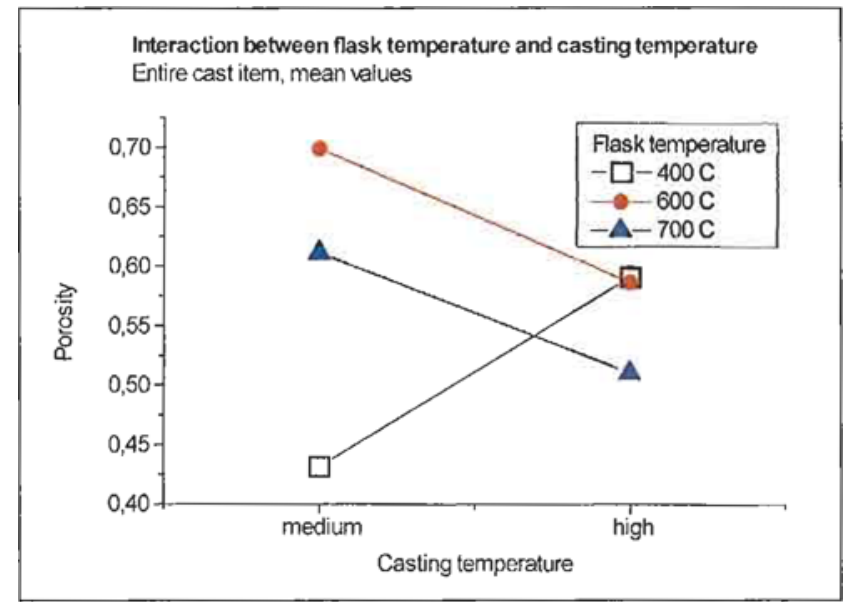

Figure 11 Interaction between flask temperature and casting temperature

This diagram shows the mean values obtained with all the alloys investigated. Casting temperature is given as a level because its absolute value depends on the type of alloy. 'Medium' means about $200^{\circ} \mathrm{C}$ above liquidus temperature, 'high' is about $300^{\circ} \mathrm{C}$ above. The low level could not be taken into account. Some alloys were not castable at this level.

Figure 11 indicates that in general a moderate casting temperature and relatively low flask temperature as well as high levels of both temperatures give a minimum of porosity. Intermediate values are detrimental. However, this average result is not true in 
every case. The two $14 \mathrm{ct}$ alloys under investigation behave somewhat differently, and the only possible conclusion in this case is that a flask temperature of $600^{\circ} \mathrm{C}$ is unfavourable. High casting and flask temperatures give a good result. The same result can be achieved with a lower flask temperature, almost independent of casting temperature.

\section{EFFECTS OF SUPERCOOLING}

The temperature difference between the true liquidus temperature and the onset of solidification at casting is defined as supercooling. Due to retarded nucleation, supercooling shows positive values under real casting conditions. This means that the metal is still completely liquid at a temperature below its real liquidus point.

In the course of the investigation supercooling was measured in several cases. A correlation between porosity and supercooling proved to be very likely. The magnitude and even the tendency seem to be strongly dependent on the alloy, the flask temperature and, to a lesser extent, casting temperature. Due to the limited number of measured values and serious experimental difficulties, a definitive and general statement cannot be given. Fairly reliable values were obtained with $18 \mathrm{ct}$ pink gold. The results indicate that increasing supercooling decreases shrinkage porosity.

\section{CONCLUSIONS}

The analysis of the results of a great number of trials has revealed the influence of some selected factors on shrinkage porosity. Only with the use of statistical methods could useful statements be obtained from widely scattered values. The most important factors studied were alloy composition (especially the copper to precious metals ratio) and the flask temperature. The casting temperature has a less pronounced effect.
Many other factors, including shape factors, have been neglected in this paper and should be considered separately.

Apart from the above, supercooling has emerged as a very significant factor controlling shrinkage porosity, due to its effect on nucleation. On the one hand, nucleation is influenced by tangible factors (eg flask temperature), but on the other it is a stochastic process which introduces a chaotic component in the casting process. A small accidental delay in nucleation influences the crystallization process, the microstructure and the porosity in an unforeseen way. This chaotic effect is seen as almost certainly the basic cause of the great scattering of the results in many of our investigations of shrinkage porosity.

\section{ACKNOWLEDGMENT}

We thank the World Gold Council (WGC) for supporting the project.

The publication is based on a presentation given at the Santa Fe Symposium and published in 'The Proceedings, The Symposium on Jewelry Manufacturing Technology 1996' (Ed. D. Schneller, Met-Chem Research Inc).

\section{ABOUT THE AUTHOR}

The author is head of the Analytical and Metallurgy Department at the German Precious Metals Research Institute (Forschungsinstitut für Edelmetalle und Metallchemie [FEM]), Schwäbisch Gmünd, Germany. He has conducted extensive research into the precious metals and their applications, and has made significant contributions to our current understanding of the investment casting of carat gold jewellery alloys and the causes of defect formation. He has published numerous articles, including several in Gold Bulletin and Gold Technology. 